\title{
Validation of population-specific Mini-Nutritional Assessment with its long-term mortality-predicting ability: results of a population-based longitudinal 4-year study in Taiwan
}

\author{
Alan C. Tsai*, Shu-Fang Yang and Jiun-Yi Wang \\ Department of Healthcare Administration, Asia University, 500 Liufeng Road, Wufeng, Taichung 41354, Taiwan
}

(Received 1 June 2009 - Revised 11 January 2010 - Accepted 12 January 2010 - First published online 1 March 2010)

Nutrition is a key element in geriatric health, and nutritional screening/assessment is a key component of comprehensive geriatric evaluation The study aimed to validate the Mini Nutritional Assessment Taiwan version-1 (MNA-T1) which adopted population-specific anthropometric cut-points, and version-2 (MNA-T2) which replaced BMI with mid-arm and calf circumferences in the scale for predicting the nutritional status of elderly Taiwanese. Using data of a population-representative longitudinal study of 2802 Taiwanese aged 65 years or older, the study graded the nutritional status of each subject with the original and both modified versions at baseline, analysed their hospital length of stay, the Activities of Daily Living (ADL), the Center for Epidemiologic Studies Depression Scale (CES-D) and life-satisfaction scores at baseline and end of 4 years, and tracked their survival during the period. Results showed that both modified versions had superior predictive abilities compared with the original MNA, and their graded scores correlated better with hospital length of stay, and ADL, CES-D and life-satisfaction scores. Both modified versions were effective in predicting follow-up mortality risk. The relative mortality risk was about 7 times for those rated malnourished and 2.5 times for those rated at risk of malnutrition compared with those who were rated normal at baseline by the two modified versions. These results suggest that both of the modified versions are effective in predicting the nutrition and health statuses of Taiwanese elderly and would serve to validate the predictive ability of the two modified versions. The MNA-T2, which requires no BMI, can make routine nutritional screening/ assessment an easier task.

Nutritional assessment: Longitudinal studies: Mortality: Validation

Nutrition is a key element in geriatric health, and nutritional screening/assessment is a key component in comprehensive geriatric evaluation. Timely screening can help detect early signs of nutritional imbalance and enable intervention to prevent emerging nutritional problems. The Mini Nutritional Assessment (MNA) is one of the most widely used and studied nutrition screening/assessment tools. It was developed and validated with clinical data of patients in Europe and USA in the 1990s for grading the nutritional risk of older adults in Western countries ${ }^{(1-3)}$. Although the tool has been used to assess the nutritional status of non-Caucasian elderly such as the Japanese ${ }^{(4,5)}$, for the best results, it should be modified according to population-specific cultural and anthropometric features in order to maintain content equivalency of the tool whenever it is applied to a population different from that for which it was designed ${ }^{(6,7)}$.

For this reason, we have modified the MNA according to Taiwanese dietary habits and anthropometric features ${ }^{(8-12)}$. Due to differences in dietary habit, we have slightly altered the questions dealing with protein intake by emphasising more on the frequency of consumption rather than number of servings. We have also adopted the Taiwanese-specific anthropometric cut-points which were derived from a dataset of a study representing Taiwanese aged 65 years or older ${ }^{(8)}$. A version with these modifications (called MNA-Taiwan version-1, MNA-T1) has been shown to improve the ability of the tool in grading the nutritional status and predicting the follow-up mortality risk of the Taiwanese elderly ${ }^{(13)}$.

Because of the difficulty in correctly measuring weight and height of frail elderly and also our belief that mid-arm circumference (MAC) and calf circumference (CC) are better indicators of health status than BMI for older elderly, we have also attempted to test a modified scale without involving $\mathrm{BMI}^{(14)}$. We displaced BMI from the scale and redistributed the assigned BMI score ( 3 points) to MAC and CC (1 point to $\mathrm{MAC}$ and 2 to $\mathrm{CC}$ ) to create a new version called the MNA-Taiwan version-2 (MNA-T2). The rationale for the modifications was based on the fact that MAC and $\mathrm{CC}$, in addition to reflecting subcutaneous body fat, also reflect lean body mass and functional status ${ }^{(14)}$, and thus can reflect overall functional status better than $\mathrm{BMI}^{(15,16)}$. The reassigning of the score was also based on the consideration that $\mathrm{CC}$

Abbreviations: ADL, Activities of Daily Living; CC, calf circumference; CES-D, Center for Epidemiologic Studies Depression Scale; MAC, mid-arm circumference; MNA-O, Mini Nutritional Assessment Taiwan modified original version; MNA-T1, Mini Nutritional Assessment Taiwan modified version-1; MTA-T2, Mini Nutritional Assessment Taiwan modified version-2.

* Corresponding author: Dr Alan C. Tsai, fax +886 4 2332, email atsai@umich.edu 
reflects functional status better than MAC except during the very late stage of functional decline ${ }^{(7)}$. This modified version has been shown to at least maintain and in many cases improve the predictive ability of the scale in elderly residing in the community, care centres and nursing homes, as well as in patients with mental disorders or on haemodialysis ${ }^{(9-12)}$. Although results from all these studies suggest that MAC and $\mathrm{CC}$ can substitute BMI in the scale without compromising the grading ability of the scale, the predictive ability of these two modified tools has yet to be tested with a more robust standard.

In developing nutrition assessment tools, we are always confronted with the difficulty of selecting a universally accepted 'gold standard' for validating a tool. Long-term survivability, although not a 'gold standard', can most probably be considered a robust standard. Thus, in the present study we attempted to use this indicator to validate the grading ability of the two modified versions of the MNA, MNA-T1 and MNA-T2.

\section{Methods}

Data of the present study were from the 'Survey of Health and Living Status of the Elderly in Taiwan' (SHLSET), a population-based longitudinal cohort study initiated in 1989 aimed to gain an understanding of the role of socio-economic, environmental, lifestyle and healthcare parameters on health, wellbeing and quality of life of older Taiwanese ${ }^{(17-19)}$. Participants were selected originally from non-institutionalised citizens according to a multi-stage national probability sampling process. Subjects in the cohort were interviewed every 2-4 years for specific purposes. A total of five rounds of interviews (in 1989, 1993, 1996, 1999 and 2003) were completed between 1989 and 2003. Data up to the 2003 survey are available for research studies. All five surveys contained a core component but each contained additional components for specific purposes. The 1999 survey contained a special component for assessing the nutritional status and that was the basis for our choosing the 1999 dataset as baseline for the present investigation. The 2003 survey was the latest dataset available and thus served as the end-point of the present study.

In each survey, trained interviewers accompanied by local health workers conducted in-home, face-to-face interviews. If a participant was unable or unavailable to have an interview, a proxy respondent, usually a close relative or caregiver of the participant was interviewed. The 1999 survey involved all 4915 remaining participants in the cohort and the youngest was aged 53 years. Of the 4915 participants, 4440 were successfully traced and interviewed for the 2003 survey ${ }^{(18,19)}$. Although the original sample (in 1989) included only non-institutional individuals, by 1999 (baseline of the present study) some were living in long-term care facilities. The present study included all available (2802) participants who were aged 65 years or older in 1999. Mortality data were based on records maintained by the survey study, but also confirmed by records of the National Health Insurance Program, or the National Household Registration.

Baseline nutritional status of each subject was evaluated with the original (MNA-O) and two modified versions (MNA-T1 and MNA-T2) of the MNA. The MNA-T1 was essentially the same as the original version, except it adopted population-specific anthropometric cut-points ${ }^{(9)}$. The MNA-T2 omitted the BMI question and redistributed the weighted score of BMI to the MAC and CC items in the scale $^{(14)}$. The study also analysed self-reported number of falls, hospital length of stay, times stayed in long-term care institutions, functional status (Activities of Daily Living (ADL) scale ${ }^{(20)}$ (degree of dependency)), psychological stress (Center for Epidemiologic Studies Depression Scale $(\mathrm{CES}-\mathrm{D})$ score $^{(21)}$ and life satisfaction ${ }^{(22)}$, both at baseline and end-point.

Spearman's correlation analysis was performed to determine the strength of the relationship between subjects' MNA scores and hospital length of stay, number of emergency visits and ADL status in 1999 and 2003. Cox regression analysis was performed to determine the significance of ageand sex-adjusted hazard ratios (relative mortality risks) among groups classified with various versions of the MNA. Age- and sex-adjusted survival curves were also shown to demonstrate differences in survival rates among these groups.

All statistical analyses were performed with SPSS (version 12.0; SPSS, Inc., Chicago, IL, USA). Statistical significance for all analyses was evaluated at $\alpha=0.05$.

The present study was conducted according to the guidelines laid down in the Declaration of Helsinki and all procedures involving human subjects were approved by government-appointed representatives. Written informed consent was obtained from all subjects. The anonymity of the subjects was preserved at all times.

\section{Results}

Table 1 shows the characteristics of subjects. Over half of the participants (59\% men and 54\% women) were aged 65-74 years; $35 \%$ men and 39\% women were aged 75-84 years and the rest, $6 \%$ men and $8 \%$ women, were aged 85 years or older. The majority of participants $(69 \%$ of men and $92 \%$ of women) had had 6 years or less of formal education. Approximately $37 \%$ of men and only $4 \%$ of women were current smokers and $36 \%$ of men and $3 \%$ of women were past smokers; $26 \%$ of men and only $4 \%$ of women drank alcohol one time or more per month; and $7 \%$ of men and $1 \%$ of women were current betel-nut chewers and about $5 \%$ of men and $4 \%$ of women were past chewers. Approximately $60 \%$ of men and $48 \%$ of women reportedly exercised at least three times per week; the rest two times or less per week. Roughly $15 \%$ of men and $24 \%$ of women had had a fall one or more times, $24 \%$ of men and $19 \%$ of women had had hospitalisation for $\geq 1 \mathrm{~d}$, and $2.1 \%$ of men and $1.4 \%$ of women had stayed in care-homes during the past 12-month period.

The patterns of nutritional status graded with the three versions of the MNA are shown in Table 2. Overall, the original MNA graded $2.9 \%$ of men and $2.8 \%$ women malnourished, and $15.1 \%$ of men and $21.2 \%$ women at risk of malnutrition; the MNA-T1 graded 2.3 and $1.7 \%$ malnourished, and 12.0 and $15.6 \%$ at risk; and the MNA-T2 graded 2.0 and $1.5 \%$ malnourished and 12.3 and $14.8 \%$ at risk for men and women, respectively. With Bonferroni correction, the patterns graded with the two modified versions were significantly 
Table 1. Characteristics (\% within sex) of subjects at baseline

\begin{tabular}{|c|c|c|}
\hline Parameters & Men $(n 1534)$ & Women ( $n$ 1268) \\
\hline \multicolumn{3}{|l|}{ Age (years) } \\
\hline $65-74$ & $59 \cdot 1$ & 53.5 \\
\hline $75-84$ & 34.9 & 38.8 \\
\hline$\geq 85$ & $6 \cdot 0$ & $7 \cdot 7$ \\
\hline \multicolumn{3}{|c|}{ Formal education (years) } \\
\hline$\leq 6$ & $68 \cdot 7$ & $92 \cdot 3$ \\
\hline $7-9$ & $13 \cdot 6$ & 4.7 \\
\hline $10-12$ & 8.6 & $2 \cdot 0$ \\
\hline$\geq 12$ & $9 \cdot 1$ & 0.9 \\
\hline \multicolumn{3}{|l|}{ Smoking habit } \\
\hline Non-smoker & $27 \cdot 1$ & $92 \cdot 7$ \\
\hline Current smoker & 37.4 & 4.4 \\
\hline Past smoker & 35.5 & $2 \cdot 8$ \\
\hline \multicolumn{3}{|l|}{ Alcohol drinking } \\
\hline$<1$ time/month & $74 \cdot 1$ & $96 \cdot 1$ \\
\hline$\geq 1$ time/month & 25.9 & 3.9 \\
\hline \multicolumn{3}{|l|}{ Routine exercise* } \\
\hline$\leq 2$ times/week & $36 \cdot 9$ & 51.9 \\
\hline $3-5$ times/week & $8 \cdot 3$ & 11.9 \\
\hline$\geq 6$ times/week & $52 \cdot 1$ & $36 \cdot 2$ \\
\hline \multicolumn{3}{|c|}{ Betel nut-chewing habit } \\
\hline Non-chewer & 88.4 & 94.6 \\
\hline Current chewer & $7 \cdot 1$ & $1 \cdot 2$ \\
\hline Past chewer & 4.5 & $4 \cdot 2$ \\
\hline \multicolumn{3}{|c|}{ Number of falls during last 12 months } \\
\hline 0 & 85.5 & $76 \cdot 1$ \\
\hline 1 & $11 \cdot 2$ & $18 \cdot 6$ \\
\hline$\geq 2$ & 3.3 & $5 \cdot 3$ \\
\hline \multicolumn{3}{|c|}{ Number of hospitalisations during last 12 months } \\
\hline 0 & $76 \cdot 2$ & 80.9 \\
\hline 1 & $16 \cdot 3$ & $12 \cdot 6$ \\
\hline$\geq 2$ & 7.5 & 6.4 \\
\hline \multicolumn{3}{|c|}{ Length of hospital stay (d) } \\
\hline 0 & $76 \cdot 3$ & $80 \cdot 9$ \\
\hline $1-6$ & 5.9 & 4.4 \\
\hline$\geq 7$ & $17 \cdot 8$ & $14 \cdot 8$ \\
\hline \multicolumn{3}{|c|}{ Duration of stay in old-age care-homes (d) } \\
\hline 0 & 97.9 & $98 \cdot 7$ \\
\hline $1-30$ & 0.3 & 0.2 \\
\hline$>30$ & 1.8 & 1.2 \\
\hline
\end{tabular}

* $\geq 30$ min per session.

different from that graded with the original MNA (both $P<0.001$ ), whereas the distributions between the two modified versions were not significantly different from each other $(Z=-2.45 ; P>0.017)$. The proportions graded malnourished or at risk of malnutrition increased with age.

The follow-up 4-year mortality rates were 70.9, 37.9 and $15.3 \%$ for elderly rated malnourished, at risk of malnutrition and normal, respectively, according to the MNA-O; 73.7, 42.4 and $16.2 \%$, respectively, according to the MNA-T1; and $77.6,43.5$ and $16.2 \%$, respectively, according to the MNA-T2 (Table 3). For elderly graded malnourished, the mortality rate for the first follow-up year was high, nearly $37 \%$ for the original scale, and 45.6 and $38.8 \%$ for the two modified versions, respectively; the rate went down to about $20 \%$ for the next 3 years. For individuals rated at risk of malnutrition, the follow-up year 1 mortality rates were 11.4 , 12.8 and $15.1 \%$ according to the three respective versions. The yearly rate stayed above $10 \%$ for all three groups for the next 3 years. For individuals rated normal at baseline, mortality rates increased gradually, from slightly above 3 to $4-5 \%$ during the next 4 years for all three versions.
Table 4 shows the Spearman's correlation coefficients $(r)$ of the MNA scores with the major health parameters evaluated at baseline (1999) and end-point (2003), respectively. The scores of all three versions of the MNA were significantly correlated with self-view health status, ADL score, CES-D score, lifesatisfaction score and hospital length of stay in 1999 and 2003, respectively. The strengths of all correlations were stronger with all indicators examined in 1999 than in 2003. Also, among the three MNA versions, the MNA-T2 showed the strongest correlation with each of these health parameters, followed by the MNA-T1, and the least with the original version in both years.

Figure 1 shows the age- and sex-adjusted survival curves and Cox regression analysis of the relative mortality risk. The survival curves among the three predicted nutritional categories were significantly different from each other in all three versions. The relative mortality risk was approximately 7 times for those rated malnourished and 2.5 times for those rated at risk of malnutrition compared with those rated normal according to Cox regression analysis adjusted for age and sex.

\section{Discussion}

Results of the present population-based 4-year longitudinal study show that the MNA is effective not only in grading the nutritional status but also in predicting the long-term mortality risk of older Taiwanese adults. Elderly who were rated malnourished or at risk of malnutrition had greatly increased risk of follow-up death compared with those who were rated normal nutritionally. During the first year following the rating, the risk of death was about 15-fold for those who were rated malnourished and 4- to 5-fold for those who were rated at risk compared with those who were rated normal, according to the two modified versions.

\section{Grading the nutritional status}

The strength of relationships between the MNA scores with several key health indicators including self-view health status, ADL score, CES-D score, life-satisfaction score and hospital length of stay suggests that among the three MNA versions, the MNA-T2 is the most capable in reflecting the health status of an individual, followed by the MNA-T1 and then the original MNA. These relationships lasted, although got weaker, 4 years after the rating. The original MNA rated significantly greater proportions of subjects malnourished or at risk of malnutrition compared with the two modified versions (MNA-T1 or MNA-T2), whereas the two modified versions produced similar results. It is understandable that the original version would rate greater proportions of elderly malnourished or at risk of malnutrition because it was designed for Westerners who usually have higher anthropometric cut-points compared with Eastern Asians. By adopting the population-specific anthropometric cut-points, the MNA-T1 corrected the population-related anthropometric bias. The MNA-T2, which further substituted the BMI item with expanded scores of MAC and CC items, appeared to have further improved the grading ability of the tool.

A tool without BMI (thus requiring no weight and height measurements) is greatly needed for individuals in less 
Table 2. Distribution of baseline nutritional status graded with the original or modified versions of the Mini-Nutritional Assessment (MNA) stratified by age ( $n$ 2802)

(Percentages)

\begin{tabular}{|c|c|c|c|c|c|c|c|c|}
\hline \multirow{3}{*}{$\begin{array}{l}\text { Age range (years)... } \\
\text { MNA versions }\end{array}$} & \multicolumn{8}{|c|}{ Distribution (\%) by age range ${ }^{*}$} \\
\hline & \multicolumn{2}{|c|}{$65-74(n 1584)$} & \multicolumn{2}{|c|}{$74-84$ ( $n$ 1028) } & \multicolumn{2}{|c|}{$>85(n 190)$} & \multicolumn{2}{|c|}{ All (n 2802) } \\
\hline & Men & Women & Men & Women & Men & Women & Men & Women \\
\hline \multicolumn{9}{|l|}{ MNA-O } \\
\hline Malnourished & $2 \cdot 2$ & 1.3 & $3 \cdot 2$ & 3.5 & $7 \cdot 6$ & $9 \cdot 2$ & 2.9 & $2 \cdot 8$ \\
\hline At risk & $12 \cdot 8$ & 14.9 & 17.4 & $25 \cdot 8$ & $25 \cdot 0$ & 41.8 & $15 \cdot 1$ & $21 \cdot 2$ \\
\hline Normal & $85 \cdot 0$ & $83 \cdot 8$ & 79.5 & $70 \cdot 7$ & $67 \cdot 4$ & 49.0 & $82 \cdot 0$ & $76 \cdot 0$ \\
\hline \multicolumn{9}{|l|}{ MNA-T1 } \\
\hline Malnourished & 1.7 & 0.6 & $2 \cdot 8$ & $2 \cdot 4$ & 6.5 & $5 \cdot 1$ & $2 \cdot 3$ & $1 \cdot 7$ \\
\hline At risk & 9.7 & $10 \cdot 6$ & 14.4 & $18 \cdot 9$ & $20 \cdot 7$ & 33.7 & $12 \cdot 0$ & $15 \cdot 6$ \\
\hline Normal & 88.6 & 88.8 & $82 \cdot 8$ & $78 \cdot 7$ & $72 \cdot 8$ & $61 \cdot 2$ & $85 \cdot 7$ & $82 \cdot 7$ \\
\hline \multicolumn{9}{|l|}{ MNA-T2 } \\
\hline Malnourished & 1.7 & 0.4 & 1.9 & $2 \cdot 0$ & $5 \cdot 4$ & $6 \cdot 1$ & $2 \cdot 0$ & 1.5 \\
\hline At risk & $9 \cdot 6$ & 10.5 & $15 \cdot 1$ & $17 \cdot 9$ & $22 \cdot 8$ & 29.6 & $12 \cdot 3$ & $14 \cdot 8$ \\
\hline Normal & $88 \cdot 7$ & $89 \cdot 1$ & $83 \cdot 0$ & $80 \cdot 1$ & $71 \cdot 7$ & $64 \cdot 3$ & $85 \cdot 7$ & $83 \cdot 7$ \\
\hline
\end{tabular}

developed countries where bathroom scales are not a common item. An attempt to construct a BMI-free nutritional assessment for the Chinese elderly was attempted by Woo et al. ${ }^{(23)}$. Using the MNA as a guide, the group developed the Chinese Nutrition Screen (CNS) without involving any anthropometric indicators. However, perhaps because of the lack of anthropometric measures, the CNS had a moderate predictive ability, with an overall $\kappa$ coefficient of 0.5 based on a two-level (normal or at risk of malnutrition) rating made by two physicians. The CNS can identify about $90 \%$ of individuals considered normal but only about $60 \%$ of individuals considered at risk of malnutrition.

Table 3. Follow-up yearly or 4-year mortality rates classified according to nutritional status graded with the original or modified versions of the Mini-Nutritional Assessment (MNA) at baseline ( $n$ 2802)

(Number of subjects and percentages)

\begin{tabular}{|c|c|c|c|c|c|c|c|c|c|}
\hline & \multicolumn{3}{|c|}{ Malnourished } & \multicolumn{3}{|c|}{ At risk } & \multicolumn{3}{|c|}{ Normal } \\
\hline & Deaths & $n \dagger$ & $\%$ & Deaths & $n \dagger$ & $\%$ & Deaths & $n \dagger$ & $\%$ \\
\hline \multicolumn{10}{|c|}{ Year 1 (1999-2000) } \\
\hline MNA-O & 29 & 79 & $36 \cdot 8$ & 57 & 501 & 11.4 & 68 & 2222 & $3 \cdot 1$ \\
\hline MNA-T1 & 26 & 57 & $45 \cdot 6$ & 49 & 382 & $12 \cdot 8$ & 79 & 2363 & $3 \cdot 3$ \\
\hline MNA-T2 & 19 & 49 & $38 \cdot 8$ & 57 & 377 & $15 \cdot 1^{*}$ & 78 & 2376 & $3 \cdot 3$ \\
\hline \multicolumn{10}{|c|}{ Year $2(2000-1)$} \\
\hline MNA-O & 12 & 50 & 24 & 46 & 444 & $10 \cdot 4$ & 81 & 2598 & $3 \cdot 1$ \\
\hline MNA-T1 & 6 & 31 & $19 \cdot 4$ & 43 & 333 & $12 \cdot 9$ & 90 & 2284 & 3.9 \\
\hline MNA-T2 & 9 & 30 & 30 & 39 & 320 & $12 \cdot 2$ & 91 & 2298 & 4 \\
\hline \multicolumn{10}{|c|}{ Year 3 (2001-2) } \\
\hline MNA-O & 9 & 38 & 23.7 & 50 & 398 & $12 \cdot 6$ & 94 & 2517 & 3.7 \\
\hline MNA-T1 & 6 & 25 & 24 & 42 & 290 & 14.5 & 105 & 2194 & 4.8 \\
\hline MNA-T2 & 7 & 21 & $33 \cdot 3$ & 38 & 281 & $13 \cdot 5$ & 108 & 2207 & 4.9 \\
\hline \multicolumn{10}{|c|}{ Year $4(2002-3)$} \\
\hline MNA-O & 6 & 29 & $20 \cdot 7$ & 37 & 348 & $10 \cdot 6$ & 97 & 2423 & 4 \\
\hline MNA-T1 & 4 & 19 & $21 \cdot 1$ & 28 & 248 & 11.3 & 108 & 2089 & $5 \cdot 2$ \\
\hline MNA-T2 & 3 & 14 & $21 \cdot 4$ & 30 & 243 & $12 \cdot 3$ & 107 & 2099 & $5 \cdot 1$ \\
\hline \multicolumn{10}{|c|}{4 years $(1999-2003)$} \\
\hline MNA-O & 56 & 79 & $70 \cdot 9$ & 190 & 501 & 37.9 & 340 & 2222 & $15 \cdot 3$ \\
\hline MNA-T1 & 42 & 57 & 73.7 & 162 & 382 & $42 \cdot 4$ & 382 & 2363 & $16 \cdot 2$ \\
\hline MNA-T2 & 39 & 49 & $77 \cdot 6$ & 164 & 377 & $43 \cdot 5 c$ & 384 & 2376 & $16 \cdot 2$ \\
\hline
\end{tabular}

MNA-O, MNA Taiwan modified original version; MNA-T1, MNA Taiwan modified version-1; MTA-T2, MNA Taiwan modified version-2.

${ }^{*}$ Significantly different from the respective MNA-O group ( $\chi^{2}$ test).

$\dagger n$ in category. 
Table 4. Spearman's correlation coefficients $(r)$ of the Mini-Nutritional Assessment (MNA) scores with health indicators at baseline (1999) and end-point (2003)

\begin{tabular}{|c|c|c|c|}
\hline Variables & MNA-O & MNA-T1 & MNA-T2 \\
\hline \multicolumn{4}{|l|}{1999 (n 2802) } \\
\hline Self-view health status & $0.409^{\star \star \star}$ & $0.437^{\star \star \star *}$ & $0.457^{\star \star \star}$ \\
\hline ADL score & $0.421^{\star \star \star}$ & $0.430^{\star \star \star}$ & $0.436^{\star \star \star}$ \\
\hline CES-D score & $-0.442^{\star \star \star}$ & $-0.471^{\star \star \star}$ & $-0.472^{\star \star \star}$ \\
\hline Life satisfaction score & $0 \cdot 313^{\star \star \star}$ & $0.324^{\star \star \star}$ & $0.327^{\star \star \star}$ \\
\hline $\begin{array}{l}\text { Hospital length of stay } \\
\text { (d/year) }\end{array}$ & $-0 \cdot 256^{\star \star \star}$ & $-0.278^{\star \star \star}$ & $-0 \cdot 290^{\star \star \star}$ \\
\hline \multicolumn{4}{|l|}{$2003(n$ 2124) } \\
\hline Self-view health status & $0.221^{\star \star \star}$ & $0.252^{\star \star \star}$ & $0.270^{\star \star \star}$ \\
\hline ADL score & $0.256^{\star \star \star}$ & $0.279^{\star \star \star}$ & $0.293^{\star \star \star}$ \\
\hline CES-D score & $-0.263^{\star \star \star}$ & $-0.278^{\star \star \star}$ & $-0.296^{\star \star \star}$ \\
\hline Life satisfaction score & $0 \cdot 192^{\star \star \star}$ & $0 \cdot 217^{\star \star \star}$ & $0.232^{* \star \star}$ \\
\hline $\begin{array}{l}\text { Hospital length of stay } \\
\text { (d/year) }\end{array}$ & $-0.070^{\star \star \star}$ & $-0.089^{\star \star *}$ & $-0 \cdot 104^{\star \star \star}$ \\
\hline
\end{tabular}

MNA-O, MNA Taiwan modified original version; MNA-T1, MNA Taiwan modified version-1; MTA-T2, MNA Taiwan modified version-2; ADL, Activities of Daily Living; CES-D, Center for Epidemiologic Studies Depression Scale. *** $P<0.001$.

\section{Predicting survivability}

The present study has demonstrated that the MNA-T2 is at least equal to and perhaps slightly better than the MNA-T1 in predicting 4-year follow-up mortality in this populationbased sample. It suggests that the MNA, in addition to being effective in rating the nutritional status, is also effective in predicting long-term mortality risk of elderly in general. The ability of the MNA to predict mortality risk has been observed in a few smaller-scale or shorter-term studies ${ }^{(14,24,25)}$. Beck et al. ${ }^{(24)}$ observed a mortality risk of $49 \%$ v. $17 \%$ for elderly at risk of malnutrition compared with those who were rated normal in the 'Survey in Europe of Nutrition in the Elderly' (SENECA) Study whereas Persson et al. ${ }^{(25)}$ observed a $40 \%$ mortality risk in 1 year and $80 \%$ in 3 years $v$. a mortality of $20 \%$ in 1 year and $50 \%$ in 3 years in eighty-three geriatric (aged 83 (SD 7) years) acute in-patients rated malnourished $v$. those who were well nourished. In an earlier study we have shown that the MNA-T1 is effective in predicting follow-up 6-month mortality risk in nursing home elderly ${ }^{(14)}$.

Malnutrition increases the risk of mortality and is also an independent predictor of mortality in older adults ${ }^{(26,27)}$. In older adults, undernutrition is closely tied to high mortality risk. Undernutrition weakens immunity and increases disease and mortality risk. On the other hand, morbidity and impaired mobility can aggravate the development of malnutrition, forming a vicious cycle. Thus, the ability to predict longterm mortality risk, perhaps, can be considered a robust test for validating a nutritional assessment tool.

\section{Relationship of Mini Nutritional Assessment score with other indicators}

The results of the present study also demonstrate a clear association of the graded nutritional status with several health-related indicators. The proportion of elderly malnourished and at risk of malnutrition increased with age. Results rated with the MNA-T2 were similar to those rated with the MNA-T1. It again suggests that the MNA-T2 is an acceptable alternative to the MNA-T1. CC and MAC can replace $\mathrm{BMI}$ in the scale without compromising the predictive ability of the tool.

The MNA-T1 rated 439 of 2802 individuals $(15.7 \%)$ undernourished (malnourished + at risk of malnutrition fractions). Among those rated as undernourished with the MNA-T1, 17.1\% (fifty-five out of 439) died within 1 year compared with $3.3 \%$ (seventy-nine out of 2363) of subjects rated normal $(17.8$ and $3.3 \%$, respectively, according to the MNA-T2). These results suggest that the MNA would be of value in serving as a screening tool for health promotion purposes at the population level. It is possible to identify the $18 \%$ high-risk individuals that account for about $50 \%$ of the total mortality risk. However, it should also be reminded that undernutrition is not the only risk of mortality in the elderly. Many other risk factors such as hypertension,
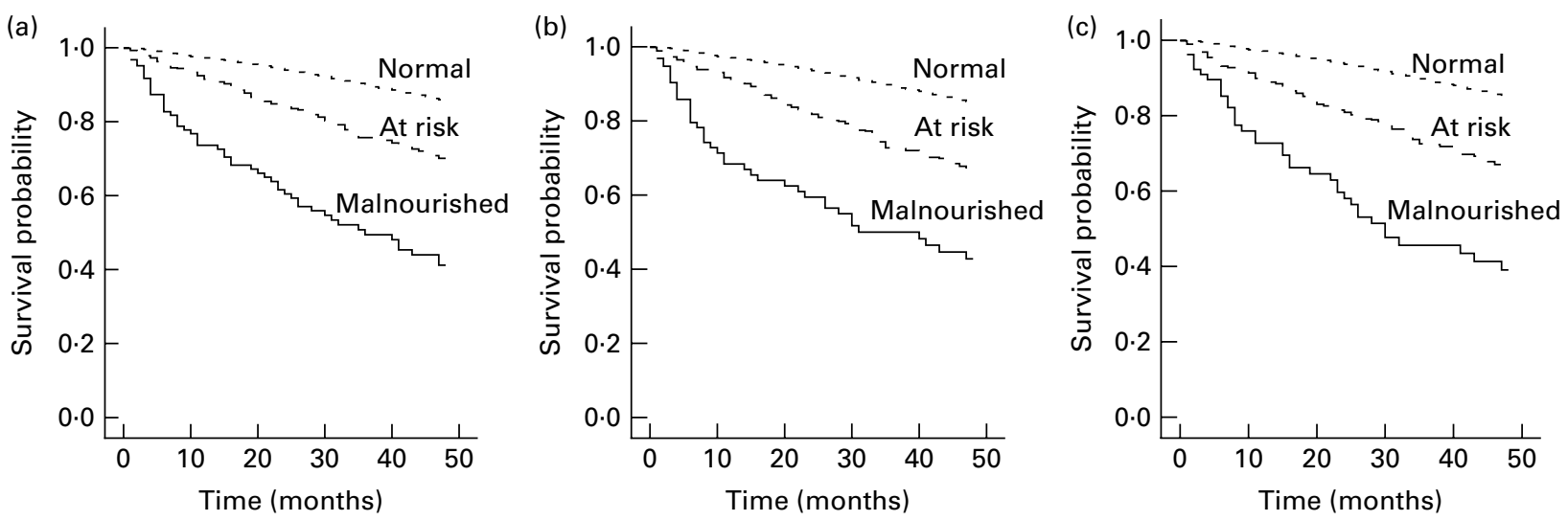

Fig. 1. Age- and sex-adjusted 4-year follow-up survival curves stratified by nutritional status graded with the original Mini-Nutritional Assessment (MNA) (a), MNA Taiwan modified version-1 (MNA-T1) (b) and MNA Taiwan modified version-2 (MNA-T2) (c), respectively. Cox regression analysis (adjusted for age and sex) showed significant differences in 4-year survival rates among the three groups in all versions (all $P<0.001$ ). The hazard ratios (relative mortality risks) for normal, at-risk and malnourished elderly were: 1.0 (reference), $2.39(95 \% \mathrm{Cl} 1.99,2.88)$ and $6.59(95 \% \mathrm{Cl} 4.94,8.79)$, respectively, graded with the original MNA; $1.0,2.55(95 \% \mathrm{Cl} 2.11,3.08)$ and $6.40(95 \% \mathrm{Cl} 4.63,8.85)$ graded with the MNA-T1 and $1.0,2.66(95 \% \mathrm{Cl} 2.20,3.21)$ and $6.79(95 \% \mathrm{Cl} 4.83,9.53)$ graded with the MNA-T2. 
type 2 diabetes, CHD, stroke, accidental injury or suicide are usually not associated with undernutrition. Other assessments would be needed to identify individuals having other types of risk.

The results of the present study again confirm the observation that the MNA not only is effective in predicting nutritional status, but can also predict general health status and the mortality risk of elderly. Elderly rated malnourished have a higher care-need ${ }^{(5)}$. Together with other indicators, the MNA might become a powerful tool for evaluating the long-term care needs of frail elderly. Although the MNA in its long-form is named an assessment tool, it probably serves the best as a screening tool because the tool does not pinpoint the exact nutritional problems nor does it identify the causes. Comprehensive examination would be needed to determine the exact nutritional problems and the intervention strategies.

\section{Limitation of the study}

Although the study involved a relatively large populationrepresentative sample of 2802 elderly subjects, it still could not adequately represent elderly with special health conditions such as kidney failure, impaired cognition or dementia. The low percentages of subjects aged older than 85 years $(6-8 \%)$ could also limit the generalisability of the results. The long-term survivability/mortality-predicting ability of the MNA was treated as a 'gold-standard' in the present study, but many deaths might not be related to malnourishment and thus could not be predicted with this tool.

\section{Conclusion}

Based on the ability of the MNA to predict long-term follow-up mortality risk and also the relationship of the MNA scores with ADL and hospital length of stay, both the MNA-T1 and -T2 have been shown to be effective in grading the nutritional status of older Taiwanese. The MNA-T2, a tool without BMI, is an acceptable alternative to the MNA-T1. It can be implemented more conveniently and efficiently and it can make periodic nutritional screening/assessment a more realistic goal in geriatric care.

\section{Acknowledgements}

The present study received no specific grant from any funding agency in the public, commercial or not-for-profit sectors. The authors wish to thank the Bureau of Health Promotion, Department of Health of Taiwan for providing the dataset for the present study.

A. C. T. designed and directed the study and was the main writer of the manuscript, S. F. Y. conducted the statistical analysis and J. Y. W. collated all statistical information.

All authors read, revised and approved the manuscript.

All authors declare that there is no conflict of interest of any kind involved in the present study.

Supplemental Appendix 1 is available online only at http:// journals.cambridge.org/action/displayJournal?jid=bjn

\section{References}

1. Guigoz Y, Vellas BJ \& Garry PJ (1994) The Mini Nutritional Assessment (MNA): a practical assessment tool for grading the nutritional state of elderly patients. In Facts and Research in Gerontology, Nutrition Supplement, pp. 15-60 [BJ Vellas, Y Guigoz, PJ Garry and JL Albarede, editors]. New York: Serdi Publishing Co.

2. Guigoz Y, Lauque S \& Villas BJ (2002) Identifying the elderly at risk for malnutrition. The Mini Nutritional Assessment. Clin Geriatr Med 18, 737-757.

3. Vellas B, Villars H, Abellan G, et al. (2006) Overview of the MNA - its history and challenge. J Nutr Health Aging 10, 456-463.

4. Kuzuya M, Kanda S, Koike T, et al. (2005) Evaluation of Mini-Nutritional Assessment for Japanese frail elderly. Nutrition 21, 498-503.

5. Izawa S, Kuzuya M, Okada K, et al. (2006) The nutritional status of frail elderly with care needs according to the Mini-Nutritional Assessment. Clin Nutr 25, 962-967.

6. Chumlea WC (1999) The state of the Mini Nutritional Assessment? Nutrition 15, 159-161.

7. Chumlea WC (2006) Is the MNA valid in different populations and across practice settings? J Nutr Health Aging 10, 524-533.

8. Tsai AC, Ho CS \& Chang MC (2007) Population-specific anthropometric cut-points improve the functionality of the Mini Nutritional Assessment (MNA) in elderly Taiwanese. Asia Pac J Clin Nutr 16, 656-662.

9. Tsai AC, Ho CS \& Chang MC (2008) Assessing the prevalence of malnutrition with the Mini Nutritional Assessment (MNA) in a nationally representative sample of elderly Taiwanese. $J$ Nutr Health Aging 12, 239-243.

10. Tsai AC \& Shih CL (2008) A population-specific Mini Nutritional Assessment can effectively grade the nutritional status of stroke rehabilitation patients in Taiwan. J Clin Nurs 18, $82-88$

11. Tsai AC, Chou YT, Chang TL, et al. (2009) A modified Mini Nutritional Assessment without BMI can effectively assess the nutritional status of neuropsychiatric patients. J Clin Nurs $\mathbf{1 8}$, 1916-1922.

12. Tsai AC, Lu SJ \& Chang TL (2008) Modified Mini Nutritional Assessment (MNA) can effectively assess the nutritional status of patients on hemodialysis. J Ren Nutr 19, 380-388.

13. Tsai AC \& Ku PY (2008) Population-specific Mini Nutritional Assessment effectively predicts the nutritional state and follow-up mortality of institutionalized elderly Taiwanese regardless of cognitive status. Br J Nutr 100, 152-158.

14. Tsai AC, Ku PY \& Tsai J-D (2008) Population-specific anthropometric cutoff standards improve functionality of the Mini-Nutritional Assessment without BMI in institutionalized elderly in Taiwan. J Nutr Health Aging 12, 696-700.

15. Luchsinger JA, Lee W, Carrasquillo O, et al. (2003) Body mass index and hospitalization in the elderly. J Am Geriatr Soc 51, $1615-1620$.

16. Reid KF, Naumova EN, Cararello RJ, et al. (2008) Lower extremity muscle mass predicts functional performance in mobility-limited elders. J Nutr Health Aging 12, 493-498.

17. Anonymous (1989) Survey of Health and Living Status of the Elderly in Taiwan: Questionnaire and Survey Design. Comparative Study of the Elderly in Four Asian Countries, Research report no. 1 (December 1989). Ann Arbor, MI: Population Studies Center University of Michigan.

18. Chang MC \& Hermalin AI (1998) 1996 Survey of Health and Living Status of the Middle Aged and Elderly in Taiwan. (A) Survey of Those 50-66 Years of Age. Elderly in Asia Research report no. 98-49, April 1998. Ann Arbor, MI: Population Studies Center University of Michigan. 
19. Chang MC \& Hermalin AI (1998) 1996 Survey of Health and Living Status of the Middle Aged and Elderly in Taiwan. (B) Survey of Those Over 67 Years of Age. Elderly in Asia Research report no. 98-50, April 1998. Ann Arbor, MI: Population Studies Center University of Michigan.

20. Katz S, Down TD, Cash HR, et al. (1970) Progress in the development of the index of ADL. Gerontologist 10, 20-30.

21. Radloff LS (1977) The CES-D scale. A self-report depression scale for research in the general population. Appl Psychol Meas 1, 385-401.

22. Diener E, Emmons RA, Larsen RJ, et al. (1985) The satisfaction with life scale. J Person Assessment 49, 71-75.

23. Woo J, Chumlea WC, Sun SS, et al. (2005) Development of the Chinese Nutrition Screen (CNS) for use in institutional settings. J Nut Health Aging 9, 203-210.
24. Beck AM, Ovesen L \& Osler M (1999) The 'Mini Nutritional Assessment' (MNA) and the 'Determine Your Nutritional Health' Checklist (NSI Checklist) as predictors of morbidity and mortality in an elderly Danish population. Br J Nutr 81, 31-36.

25. Persson MD, Brismar KE, Katzarski KS, et al. (2002) Nutritional status using Mini Nutritional Assessment and Subjective Global Assessment predict mortality in geriatric patients. J Am Geriatr Soc 50, 1996-2002.

26. Sullivan DH, Wall RC \& Lipschitz DA (1991) Protein-energy undernutrition and the risk of mortality within $1 \mathrm{y}$ of hospital discharge in a select population of geriatric rehabilitation patients. Am J Clin Nutr 53, 599-605.

27. Robertson RG \& Montagnini M (2004) Geriatric failure to thrive. Am Fam Physician 70, 343-350. 